\title{
Infected urachal sinus presenting as infraumbilical mass- A Case report and review of literature
}

\author{
Rajkumar S Yalawar ${ }^{1}$, Raghavendra Khanapur ${ }^{1}$, L S Patil ${ }^{2}$, Akil Kulkarni ${ }^{1}$ \\ ${ }^{I}$ Department of Radiology, SSIMS \& RC, Davangere, Karnataka, India. \\ ${ }^{2}$ Department of Surgical Oncology, SSIMS \& RC, Davangere, Karnataka, India.
}

\begin{abstract}
Urachal sinus is rare congenital urachal anomaly in adults develops from incomplete obliteration of fetal urachus. Usually incidental finding and remain asymptomatic unless a complication occurs. Complications includes infection, abscess, calculus, gas formation, intraperitoneal rupture, peritonitis and malignant transformation. High index of clinical suspicion is required when patient presents with pain abdomen, infraumbilical mass or umbilical discharge. Infected urachal sinus remains a diagnostic challenge due to its rare incidence and variable clinical presentations. We report a case in 37 year old female patient of infected urachal sinus presenting as infraumbilical mass, umbilical discharge and local tenderness. Two months back presented with fever, lower abdominal pain of unknown origin. Clinical suspicion of abdominal wall abscess or omphalitis were made. In our case ultrasonography and magnetic resonance imaging confirmed this rare entity. Brief discussion were made on clinical presentation, differential diagnosis, radiological findings and management options in order to prevent further complications, recurrence and malignant transformation.
\end{abstract}

Keywords: Abscess, Urachus, Urachus sinus, Umbilical discharge

\section{Introduction}

Urachus is a vestigial remnant that originates from involution of the cloaca and allantois. Urachal abnormalities result from incomplete obliteration of the foetal urachus. Since the first description by Cabriolus in 1550 , few case reports of infected urachal sinus have been reported in literature ${ }^{1}$. Usually discovered in childhood but rare in adulthood unless they present with complication. Most of the congenital urachal remnants are asymptomatic except for patent urachus. Symptomatic urachal remnants in adults remains a diagnostic challenge due to variable clinical presentation and rare incidence in general population. This article emphasis on clinical presentation, radiological diagnosis, differentials and management in a rare case of infected urachus sinus in adult.

\section{Case Report}

A 37 year-old female patient presented with a 3 days history of intermittent purulent umbilical discharge, dull lower abdominal pain and infraumbilical mass since a week. No history of dysuria or frequency of urination. Fever was observed for 3-4 days in a week for which she was on paracetamol medication. There was 4 days of hospitalization in the past for fever, lower abdominal pain and few episodes of vomiting 2 months back of unknown cause. Ultrasonography of abdomen, blood and urine reports were normal. Conservative management was done and later discharged. Recent onset of clinical presentation were evaluated. On physical examination patient is afebrile, purulent umbilical discharge and erythema (figure 1) was noted. On palpation abdomen was soft but local tenderness in infraumbilical region. Laboratory data revealed a white blood cell count of $21.1 \times 10^{3} / \mu \mathrm{L}$, hemoglobin level was $10.2 \mathrm{~g} / \mathrm{dL}$, and platelet count of $321 \times 10^{3} / \mu \mathrm{L}$. Blood biochemistry revealed sodium $138 \mathrm{mEq} / \mathrm{L}$, potassium $2.1 \mathrm{mEq} / \mathrm{L}$, chloride $89 \mathrm{mEq} / \mathrm{L}$, bicarbonate $48 \mathrm{mEq} / \mathrm{L}$, BUN $28 \mathrm{mg} / \mathrm{dL}$, creatinine $1.1 \mathrm{mg} / \mathrm{dL}$, glucose $108 \mathrm{mg} / \mathrm{dL}$, and calcium $8.3 \mathrm{mg} / \mathrm{dL}$. Urinalysis analysis and renal function were normal. A cotton swab was inserted into the opening of the wound to obtain innermost material for culture and antibiotic sensitivity. Initial management was started with broad spectrum IV antibiotic therapy. Culture of the umbilical discharge showed E. coli colonies. Treatment was started with intravenous ciprofloxacin for a week and later on switched on to tablet. Ultrasonography of abdomen shows heterogeneous inflammatory collection, echogenic calculus and few air pockets in the lower abdominal wall near the umbilicus. No obvious communication with bowel or ascites ( Figure 2). Magnetic resonance imaging confirmed the diagnosis of infected urachal sinus presenting as a abscess, $1.2 \mathrm{~cm}$ calculus and air pockets communicating with umbilicus in proximal aspect. Distal aspect showed a fibrous cord attached to bladder apex. No communication with bladder was made which was confirmed on cystoscopy. After 6 week, infraumbilical midline incision was used to excise urachus sinus and fibrous tract with a bladder cuff. The postoperative course was uneventful. Histological examination did not reveal any signs of malignancy. No recurrence after 12 month follow up. 


\section{Discussion}

Urachus is a vestigial remnant of atleast two embryonic structures cloaca and allantois. The tubular urachus normally involutes before birth and remaining as a fibrous cord in adult ${ }^{2}$. It is located in extraperitoneal space of Retzius in the anterior abdominal wall between transverse fascia and parietal peritoneum extending from anterior dome of the bladder toward the umbilicus. The urachus varies from 3 to $10 \mathrm{~cm}$ in length and 8 to $10 \mathrm{~mm}$ in diameter.

There are 3 layers histologically, an innermost layer of modified transitional epithelium similar to the urothelium, a middle layer of fibro-connective tissue and an outermost layer of smooth muscle continuing the detrusor ${ }^{3,4}$. Usually discovered in early childhood but is rare in adults. Congenital urachal anomalies are twice as common in men as in women 5 .

Urachal abnormalities result from incomplete obliteration of the foetal urachus. There are five types of urachal abnormalities: (1) patent urachus, in which the entire tubular structure fails to close (50\%); (2) urachal cyst, in which both ends of the canal close leaving an open central portion $(30 \%)$; (3) urachal sinus, which drains proximally into the umbilicus $(15 \%)$; (4) vesicourachal diverticulum, where the distal communication to the bladder persists (3-5\%) and (5) Alternating sinus, which can drain to either bladder or umbilicus ${ }^{6,7}$.

Urachal sinus abscess usually occurs by infection of mucinous secretion via the umbilicus or infection of hair follicles at periumbilical region. Umbilical-urachal sinus consists of blind dilatation of the urachus at the umbilical end and may result in periodic discharge ${ }^{8,9}$. The commonly cultured microorganisms from the pus are staphylococcus aureus, Escherichia coli, Enterococcus faecium, Proteus, Citrobacter species, Streptococcus Viridans and Fusobacterium ${ }^{6,7}$

The clinical signs and symptoms are nonspecific and variable. In most cases urachal sinus are asymptomatic unless they become infected. Clinical triad of symptoms including a tender midline infraumbilical mass, umbilical discharge and sepsis should arouse suspicion of urachal sinus ${ }^{10}$. In our case, initial clinical presentation was vague so clinicians didn't have any clue in the past. Later patient presented with lower abdominal pain and infraumbilical mass. Ultrasonography of anterior abdominal wall showed induration of infraumbilical soft tissue and suspected evolving abscess. Patient was treated with analgesic and antibiotic therapy for a 1 week. At follow up umbilical discharge and local tenderness in infraumbilical region, then high clinical suspicion of abdominal wall abscess and omphalitis was made.

Ultrasonography of abdomen was initial investigation to screen the abdominal wall and intraabdominal structures. In our case infraumbilical abscess was noted in lower abdominal wall with echogenic calculus and air foci near the umbilicus. No intraperitoneal / bowel communication was observed. No evidence of free fluid. Urinary bladder lumen was well distended. Then further evaluation was carried out. Magnetic resonance imaging (MRI) of lower abdomen shows heterogeneous infraumbilical abdominal abscess, calculus and gas pockets near to the umbilicus with opening into the umbilicus. Distally fibrous cord is traced upto the bladder apex. No abnormal soft tissue at bladder dome or cystic structure. No obvious communication with bladder lumen. MRI is the investigation of choice for better soft tissue characterization, multiplanar and radiation free. MRI of abdomen was far superior to computed tomography fistulography in demonstrating sinus, soft tissue characterization and distal attachment to bladder. It also excludes any mass lesion at bladder dome.

Omphalitis, Umbilical granuloma, patent omphalomesenteric duct, patent urachus are considered differentials of umbilical discharge in newborn and children. Urachal cyst, urachal carcinoma, umbilical hernia ulceration, abdominal abscess are some of differentials in adults ${ }^{11}$. Urachal sinus can be complicated by abscess, stone and gaseous formation as reported in our case. When urachal abscess was diagnosed, differentials like cellulitis, necrotizing fasciitis, peritonitis, acute appendicitis, hematoma, ventral or umbilical hernia and tumor lesions especially when it develops into the abdominal wall were considered ${ }^{12}$. Other reported complications in literature include rupture into the peritoneal cavity leading to peritonitis, uracho-colonic fistula and neoplastic transformation ${ }^{6}$. Malignant urachal neoplasms are also rare, representing less than $0.5 \%$ of all bladder cancers ${ }^{13}$. Urachal carcinoma predominantly manifests as adenocarcinoma ( $90 \%$ of cases) probably due to the metaplasia of the urachal mucosa into columnar epithelium followed by malignant transformation. About $34 \%$ of bladder adenocarcinomas are of urachal origin ${ }^{13,14}$. These tumors are most commonly seen in patients $40-70$ years of age and common in men ${ }^{15}$. Few cases of urachal carcinoma reported in middle of the urachus ( $6 \%$ of cases) or near the umbilical end $(4 \%)^{16}$.

Urachal remnants treatment depends on the complications. Incidental detected urachal sinus without any complication are usually removed in a single step radical excision of urachal remnant. In our case infected urachal sinus presented with complications of abscess, calculus and gas formation so aggressive treatment was done. Failure to make early diagnosis may result in poor outcome including septic shock and death. In our case effective treatment consists of appropriate antibiotics depending upon culture of organism and antibiotic sensitivity, to allow rapid resolution of adjacent inflammatory process, to limit the spread of pathogens and further reduces the later surgical risk of wound infection ${ }^{11}$. After a short follow up the patient underwent radical excision of urachal remnant with bladder cuff via open midline infraumbilical incision. In literature, 
some authors reported single stage procedure backed with appropriate antibiotic therapy or two stage procedure involving initial incision and drainage, followed by later excision of the urachal remnant with or without bladder cuff via open or laparoscopic surgical approach ${ }^{12}$. This intervention is performed to ovoid recurrence following simple drainage and to prevent developing malignant transformation.

\section{Conclusion}

Infected urachal sinus is a diagnostic challenge due to its rare incidence and nonspecific clinical presentation in adults. High index of suspicion is required to diagnosis this entity based on clinical triad of infraumbilical mass, umbilical discharge, and sepsis. In our case report we highlight basic anatomical knowledge, radiological findings and appropriate management options based on clinical presentations. We emphasis the importance of early and accurate diagnosis in order to prevent further complications, recurrence and malignant transformation.

\section{Acknowledgements}

I am thankful to Dr Ramesh Desai for guiding my research work and radiology technical staff.

Fund Support: Nil

Conflict of interest: None

\section{References}

[1]. Hsu C-C, Liu Y-P, Lien W-C, Lai T-I, Chen W-J, Wang H-P: Urachal abscess: a cause of adult abdominal pain that cannot be ignored. Am J Emerg Med 2005. 23:229-230.

[2]. Moore KL. The urogenital system. In: Moore KL, Ed. The developing human. 3rd ed. Philadelphia, Pa: Saunders, 1982; $255-297$.

[3]. N. K. Mahato, M. M. Mittal, R. Aggarwal, K. M. Munjal. "Encysted urachal abscess associated with a premalignant lesion in an adult male". Uro Today International Journal 2010. vol. 3, no. 5.

[4]. H. G. O. Mesrobian, A. Zacharias, A. H. Balcom, R.D. Cohen, "Ten years of experience with isolated urachal anomalies in children". Journal of Urology 1997. vol. 158, no. 3, pp.1316-1318.

[5]. Friedland GW, Devries PA, Matilde NM, Cohen R, Rifkin MD. Congenital anomalies of the urinary tract. In: Pollack HM, ed. Clinical urography. Philadelphia, Pa: Saunders, 1990; 559-787.

[6]. C. E. Kingsley and J. P. Nigel, "Infected urachal cyst in an adult: a case report and review of the literature," Cases Journal 2009: vol. 2, no. 6, Article ID 6422.

[7]. R. F. Spataro, R. S. Davis, and M. S. F. McLachlan, “Urachal abnormalities in the adult," Radiology 1983.Vol. 149, no. 3, pp. 659663.

[8]. Berman SM, Tolia BM, Laor E, Reid RE, Schweizerhof SP, Freed SZ. Urachal remnants in adults. Urology 1988; 31:17-21.

[9]. Blichert-Toft M, Nielsen OV. A congenital patent urachus and acquired variants. Acta Chir Scand 1971; 137:807-814.

[10]. M. M. Tiao, S. F. Ko, S. C. Huang, C. S. Shieh, C. L. Chen. "Urachal inflammatory mass mimicking an intra-abdominal tumor two years after excision of the urachal sinus in a child". Chang Gung Medical Journal 2003. Vol. 26, no. 8, pp. 598-601.

[11]. Michael S Martin, Robert M Lembo. A 17 year old boy with umbilical discharge. Pediatric Rounds. Hospital physician. May 2004; pp 19-25.

[12]. Baldassarre E, Lillaz B, Vittoria I, Pierini P: Asymptomatic chronic urachal abscess mimicking a bladder tumor. Arch Ital Urol Androl 2009, 81:251-252.

[13]. Sheldon CA, Clayman RV, Gonzalez R, Williams RD, Fraley EE. Malignant urachal lesions. Journal of Urology 1984; 131:1-8.

[14]. Ravi R, Shrivastava BR, Chandrasekhar GM, Prahlad S, Balasubramanian KV. Adenocarcinoma of the urachus. Journal of Surgical Oncology 1992; 50:201-203.

[15]. Kwok-Liu JP, Zikman JM, Cockshott WP. Carcinoma of the urachus: the role of computed tomography. Radiology 1980; 137:731734.

[16]. Beck AD, Gaudin JH, Bonham DG. Carcinoma of the urachus. Br J Urol 1970; 42:555-562.

[17]. P. Yohannes, T. Bruno, M. Pathan, R. Baltaro. "Laparoscopic radical excision of urachal sinus". Journal of Endourology 2003. Vol. 17 , no. 7, pp. $475-479$.

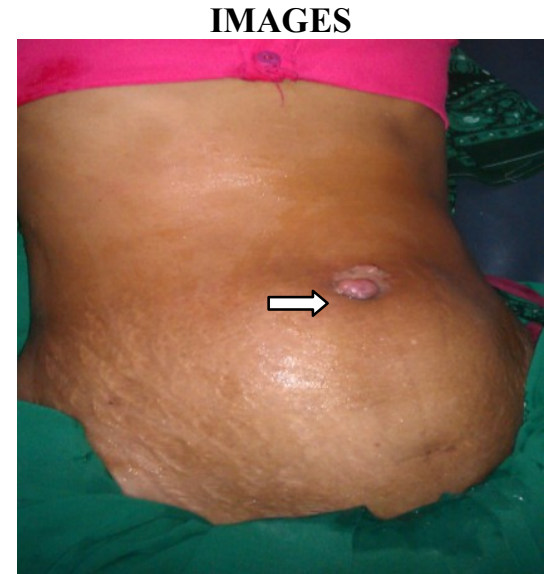

Figure 1. Infraumbilical mass with purulent umbilical discharge 


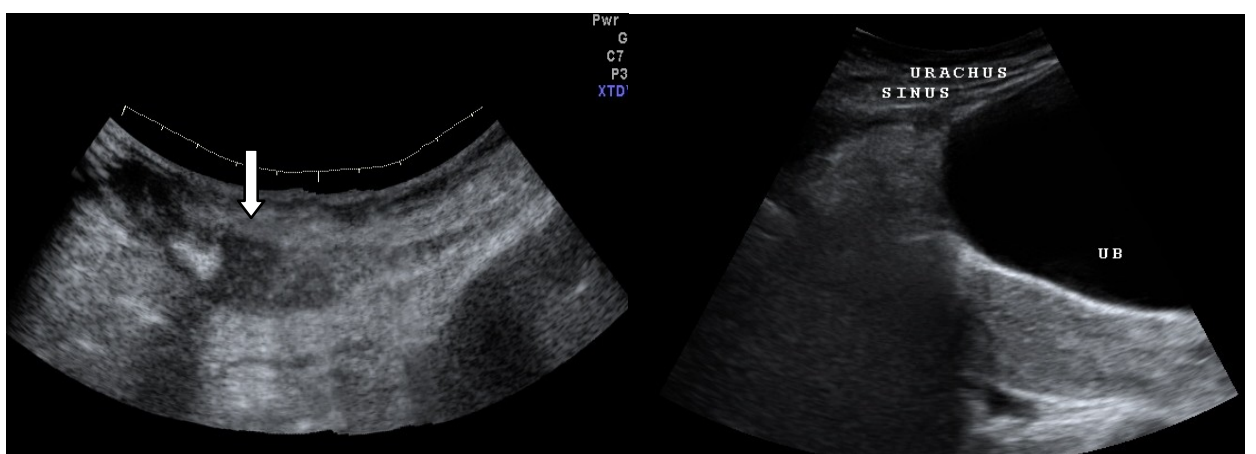

Figure 2. Ultrasonography of abdomen (A and B): A. Heterogeneous inflammatory collection, echogenic calculus and few air pockets. B. Fibrous cord like structure attached to bladder apex.

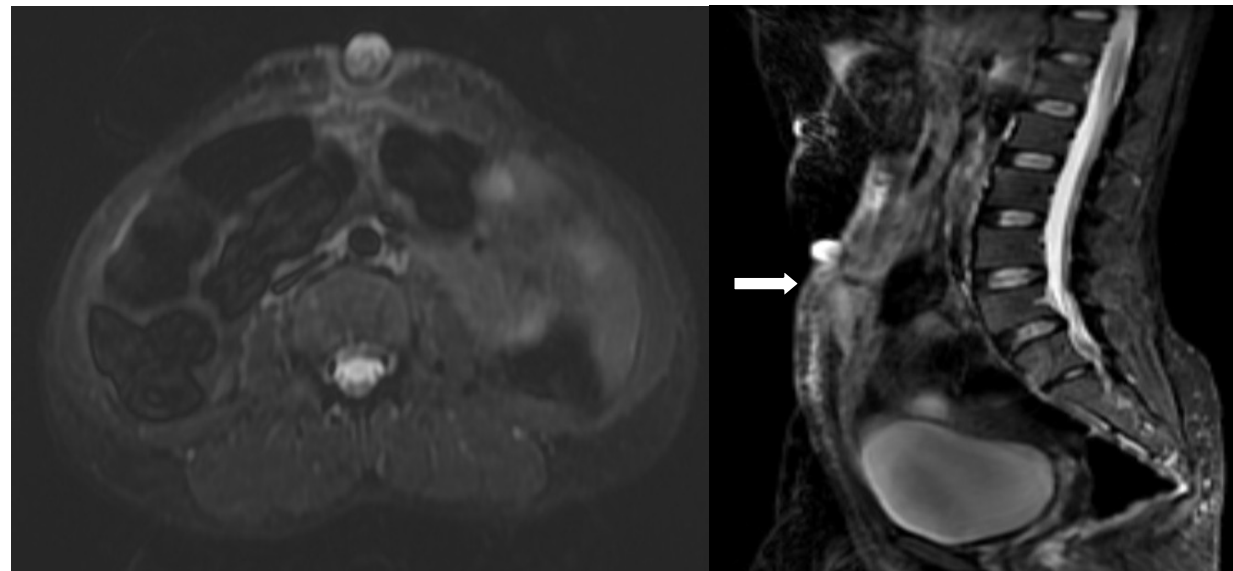

Figure 3. Short tau inversion recovery (STIR ) axial and sagittal images A and B: Heterogeneous inflammatory collection with oval hypointense calculus in the infraumbilical region opening into the umbilicus. Fibrous cord like structure in distal aspect attached to bladder apex. No communication with bladder lumen. 